
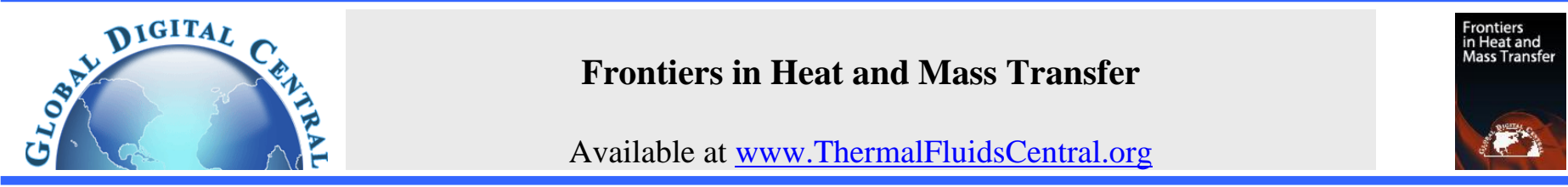

\title{
NONLINEAR CONVECTIVE TRANSPORT ALONG AN INCLINED PLATE IN NON-DARCY POROUS MEDIUM SATURATED BY A MICROPOLAR FLUID WITH CONVECTIVE BOUNDARY CONDITION
}

\author{
Ch. RamReddy ${ }^{\dagger}$, P. Naveen, and D. Srinivasacharya \\ Department of Mathematics, National Institute of Technology Warangal-506004, India.
}

\begin{abstract}
The role of nonlinear variation of density with temperature (NDT) and concentration (NDC) on the free convective flow of non-Darcy micropolar fluid over an inclined plate has been studied for the first time. In addition, the modified form of thermal slip and isothermal condition is utilized to address heat transfer phenomena in nuclear plants, textile drying, and heat exchangers, etc. The respective partial differential equations and boundary conditions are cast into a sequence of the ordinary differential equation by the local non-similarity technique. The remodeled equations are simplified numerically by applying a successive linearization method (SLM). A constructive investigation emphasizing the influence of the Biot number, inclination of angle, nonlinear convection parameters on the drag coefficient, couple stress, mass and heat transfer rates. The results of this qualitative analysis are displayed graphically and the physical significance of the pertinent parameters is discussed in detail.
\end{abstract}

Keywords: Successive linearization method, Micropolar fluid, Non-linear convection Convective boundary condition Non-Darcy porous medium.

\section{INTRODUCTION}

Free convective transport of mass and heat transfer from non-identical geometries in a porous medium has a huge thrust in geophysical and engineering industries such as moisture over reservoirs in geothermal engineering, agricultural fields, drying of porous solids etc. A comprehensive evaluation of convective heat transfer of various fluids in the nonDarcy porous medium can be seen in the textbook by Nield and Bejan (2013). Eringen (1966) initiated the theory of a micropolar fluid to describe fluids which contradict to Newton's law of viscosity like animal blood, polymeric fluids, etc. The micropolar fluid contains rigid, cylindrical elements, for instance, large dumbbell-shaped molecules, the intrinsic motion, and microstructure of fluid elements are conceptualized in this theory. The mathematical aspects of micropolar fluid theory and its applications are reported in the books by Lukaszewicz (1999) and Eremeyev et al., 2013. Further, various authors to mention few ( Murthy and Singh, 1999; Bég et al., 2008; Srinivasacharya and RamReddy, 2013 ) scrutinized the mass and heat transfer characteristics of non-Newtonian and viscous fluid flows over a different surfaces in the Forchheimer porous medium.

The foremost theories of heat transfer in porous medium concentrated its attention on the onset of thermal convection by adopting a linear relation between density and temperature. But, it is noticed by many researchers that the density temperature relationship may become nonlinear, if the difference between the ambient fluid temperature and the plate temperature is appreciably large (for more details see Barrow and Sitharamarao, 1971; Vajravelu and Sastri, 1977). Partha (2010) studied the effect of nonlinear convection in a non-Darcy porous medium and concluded that, with the increase of nonlinear concentration-temperature parameter, increases the mass and heat transfer rates rapidly in Darcy porous medium when compared with the Forchheimer model. The effects of nonlinear convection and thermophoresis in a non-Darcy porous medium have been discussed by Kameswaran et al. (2014) and concluded that the temperature and concentration boundary layer thickness reduces with enhancing values of nonlinear concentration (NDC) and temperature (NDT) parameters. Quite recently, Shaw et al. (2016) focused on the behavior of non-linear thermal convection in nanofluid flow over a stretching surface.

In the field of thermal insulation, material processing, and geothermal systems the flux or isothermal conditions are inappropriate, due to thermal stresses caused by natural convection results to damage in the nuclear reactors piping systems. To overcome this, a fixed amount of heat is supplied to the fluid through a surface so that damage caused by the continuous supply of heat transfer can be controlled. This type of analysis frequently appears in industrial and engineering processes such as transpiration cooling, material drying process, etc. Makinde (2010) analyzed the impact of convective boundary condition on hydromagnetic flow over a moving vertical plate whereas Yacob and Ishak (2012) con-

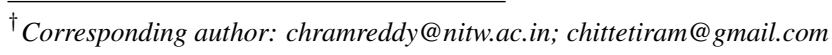


sidered micropolar fluid past stretching sheet and provided the similarity solutions. RamReddy et al. (2015) addressed convective surface boundary condition on the natural convective micropolar fluid flow along the vertical plate, the authors reported that the enhancement of Biot number leads a nonlinear growth in mass and heat transfer rates within the boundary layer(also refer the references given therein). In recent times, the influence of Joule heating and thermal radiation on MHD micropolar fluid has been discussed by Ramzan et al. (2016) by taking the partial slip and convective surface boundary conditions.

In this study, the influence of NDT and NDC parameters on natural convection flow along an inclined plate in a non-Darcy micropolar fluid under the convective surface boundary condition has been analyzed. The governing highly nonlinear system of partial differential equations is transformed to a system of nonlinear ordinary differential equations by local non-similarity procedure and then the successive linearization method is used to solve reduced boundary value problem. Hence, the physically important profiles are illustrated for representative values of the major parameters. This kind of analysis has important applications in aerosol technology, high-temperature polymeric mixtures, which are associated with temperature-concentration-dependent density.

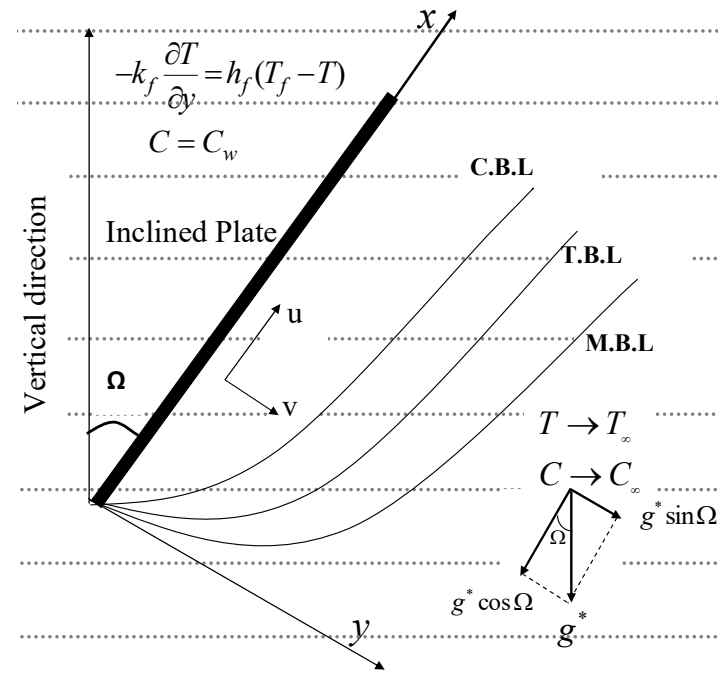

Fig. 1 Physical model

\section{MATHEMATICAL FORMULATION}

Consider a two-dimensional steady free convection flow of an incompressible micropolar fluid over a semi-infinite inclined flat plate in a nonDarcy porous medium, with an acute angle to the vertical direction and constant physical properties expect in the buoyancy term, as shown in Fig. (1). The respective distances along the plate and normal to the plate in the coordinate system are measured by $x$ and $y$. The plate is either cooled or heated from a fluid of temperature $T_{f}$ to the left by convection with $T_{f}<T_{\infty}$ relating to a cooled surface and $T_{f}>T_{\infty}$ relating to a heated surface respectively. Over the wall the solutal concentration is taken to be constant $C_{w}$ and the free stream concentration and temperature are assumed to be $C_{\infty}$ and $T_{\infty}$ respectively. Further, the temperature and concentration variation between the ambient fluid and surface of the plate assumed to be notably large, so that the NDT and NDC variations in the buoyancy force term exert a strong influence on the flow field.

By using nonlinear Boussinesq approximation and boundary layer assumptions, the flow governing equations for Forchhiemer model of the micropolar fluid are given by

$$
\frac{\partial u}{\partial x}+\frac{\partial v}{\partial y}=0
$$

$$
\begin{gathered}
\frac{\rho}{\varepsilon^{2}}\left(u \frac{\partial u}{\partial x}+v \frac{\partial u}{\partial y}\right)=\frac{1}{\varepsilon}(\mu+\kappa) \frac{\partial^{2} u}{\partial y^{2}}+\kappa \frac{\partial \omega}{\partial y}-\frac{\mu}{K_{P}} u-\frac{\rho b}{K_{P}} u^{2}+\rho g^{*} \\
{\left[\beta_{0}\left(T-T_{\infty}\right)+\beta_{1}\left(T-T_{\infty}\right)^{2}+\beta_{2}\left(C-C_{\infty}\right)+\beta_{3}\left(C-C_{\infty}\right)^{2}\right] \cos \Omega} \\
\frac{\rho j}{\varepsilon}\left(u \frac{\partial \omega}{\partial x}+v \frac{\partial \omega}{\partial y}\right)=\gamma \frac{\partial^{2} \omega}{\partial y^{2}}-\kappa\left(2 \omega+\frac{1}{\varepsilon} \frac{\partial u}{\partial y}\right) \\
u \frac{\partial T}{\partial x}+v \frac{\partial T}{\partial y}=\alpha \frac{\partial^{2} T}{\partial y^{2}} \\
u \frac{\partial C}{\partial x}+v \frac{\partial C}{\partial y}=D \frac{\partial^{2} C}{\partial y^{2}}
\end{gathered}
$$

where $\omega$ is the microrotation component in which the direction of rotating fluid element in the $x y$-plane, $(u, v)$ are the Darcian velocities. Also, $b, \varepsilon, K_{p}, g^{*}, C, T, \mu, \rho, j, \Omega, \kappa$ and $\gamma$ denotes the empirical constant, porosity, permeability, acceleration due to gravity, concentration, temperature, dynamic viscosity, density, micro-inertia density, inclination of angle, vortex viscosity and spin-gradient viscosity, respectively. Here $\beta_{0}$ and $\beta_{1}, \beta_{2}$ and $\beta_{3}$ are the first and second order expansions of thermal and solutal coefficients, respectively. Further, $D$ and $\alpha$ are the solutal and thermal diffusivities of the medium, respectively.

The subject to the boundary conditions are

$$
\begin{gathered}
\text { on } y=0: u=0, v=0, \omega=0,-k_{f} \frac{\partial T}{\partial y}=h_{f}\left(T_{f}-T\right), C=C_{w} \\
\quad \text { as } y \rightarrow \infty: \quad u=0, \quad \omega=0, \quad T=T_{\infty}, \quad C=C_{\infty}
\end{gathered}
$$

where, $k_{f}$ and $h_{f}$ indicates the thermal conductivity of the fluid and convective heat transfer coefficient, respectively.

Introducing the following dimensionless variables

$$
\left.\begin{array}{l}
\xi=\frac{x}{L}, \eta=\frac{y}{L}\left(\frac{G r}{\xi}\right)^{\frac{1}{4}}, \psi(\xi, \eta)=\frac{\mu G r^{\frac{1}{4}} \xi^{\frac{3}{4}}}{\rho} f(\xi, \eta) \\
\omega(\xi, \eta)=\frac{\mu G r^{\frac{3}{4}} \xi^{\frac{1}{4}}}{\rho L^{2}} g(\xi, \eta), \theta(\xi, \eta)=\frac{T-T_{\infty}}{T_{f}-T_{\infty}}, \\
\phi(\xi, \eta)=\frac{C-C_{\infty}}{C_{w}-C_{\infty}}
\end{array}\right\}
$$

In view of the continuity equation (1), we introduce the stream function $\psi$ by

$$
u=\frac{\partial \psi}{\partial y}, \quad v=-\frac{\partial \psi}{\partial x}
$$

Using Eqs. (7) and (8) into Eqs.(2) - (5), we get the following dimensionless momentum, angular momentum, energy and concentration equations

$$
\begin{gathered}
\frac{1}{\varepsilon}\left(\frac{1}{1-N}\right) f^{\prime \prime \prime}+\frac{3}{4 \varepsilon^{2}} f f^{\prime \prime}-\frac{1}{2 \varepsilon^{2}} f^{\prime 2}+\left(\frac{N}{1-N}\right) g^{\prime} \\
-\frac{F s}{D a} \xi f^{\prime 2}-\frac{\xi^{\frac{1}{2}}}{D a G r^{\frac{1}{2}}} f^{\prime}+\left[\theta\left(1+\alpha_{1} \theta\right)+\mathcal{B} \phi\left(1+\alpha_{2} \phi\right)\right] \cos \Omega \\
=\frac{\xi}{\varepsilon^{2}}\left(f^{\prime} \frac{\partial f^{\prime}}{\partial \xi}-f^{\prime \prime} \frac{\partial f}{\partial \xi}\right) \\
\lambda g^{\prime \prime}+\frac{3}{4 \varepsilon} f g^{\prime}-\frac{1}{4 \varepsilon} f^{\prime} g-\left(\frac{N}{1-N}\right) \mathcal{J} \xi^{\frac{1}{2}}\left(2 g+\frac{1}{\varepsilon} f^{\prime \prime}\right) \\
=\frac{\xi}{\varepsilon}\left(f^{\prime} \frac{\partial g}{\partial \xi}-\frac{\partial f}{\partial \xi} g^{\prime}\right) \\
\frac{1}{\operatorname{Pr}} \theta^{\prime \prime}+\frac{3}{4} f \theta^{\prime}=\xi\left(f^{\prime} \frac{\partial \theta}{\partial \xi}-\frac{\partial f}{\partial \xi} \theta^{\prime}\right) \\
\frac{1}{S c} \phi^{\prime \prime}+\frac{3}{4} f \phi^{\prime}=\xi\left(f^{\prime} \frac{\partial \phi}{\partial \xi}-\frac{\partial f}{\partial \xi} \phi^{\prime}\right)
\end{gathered}
$$

In the above equations, the primes represent partial derivative in respective of $\eta$ alone. Further, $N=\kappa /(\mu+\kappa),(0 \leq N<1)$ ( Cowin, 1968), $G r=\left[g^{*} \beta_{0}\left(T_{f}-T_{\infty}\right) L^{3}\right] / \nu^{2}, \nu, D a=K_{p} / L^{2}, \mathcal{B}=\beta_{2}\left(C_{w}\right.$ 
Table 1 Comparison of $-\theta^{\prime}(\xi, 0)$ for natural convection along a vertical flat plate in regular fluid when $N=0, \mathcal{B}=0, \alpha_{1}=0, \alpha_{2}=0$, $\varepsilon=1, D a \rightarrow \infty, \lambda=0, \xi=1, B i \rightarrow \infty, \Omega=0$ and $\operatorname{Pr}=1$.

\begin{tabular}{|c|c|}
\hline Bejan (2013) & Present \\
\hline 0.401 & 0.40103 \\
\hline
\end{tabular}

$\left.C_{\infty}\right) /\left[\beta_{0}\left(T_{f}-T_{\infty}\right)\right], F s=b / L, \operatorname{Pr}=\nu / \alpha, \lambda=\gamma /(j \rho \nu), \mathcal{J}=$ $L^{2} /\left(j G r^{\frac{1}{2}}\right), S c=\nu / D, \Omega, \alpha_{1}=\beta_{1}\left(T_{f}-T_{\infty}\right) / \beta_{0}$ and $\alpha_{2}=\beta_{3}\left(C_{w}-\right.$ $\left.C_{\infty}\right) / \beta_{2}$ indicates the coupling number, global thermal Grashof number, kinematic viscosity, Darcy number, Buoyancy ratio, Forchheimer number, Prandtl number, spin-gradient viscosity, micro-inertia density, Schmidt number, angle of inclination, nonlinear temperature parameter(NDT) and nonlinear concentration parameter(NDC), respectively.

The boundary conditions (6) become

$$
\begin{array}{r}
f^{\prime}(\xi, 0)=0, f(\xi, 0)=-\frac{4}{3} \xi\left(\frac{\partial f}{\partial \xi}\right)_{\eta=0}, g(\xi, 0)=0, \\
\theta^{\prime}(\xi, 0)=-B i \xi^{\frac{1}{4}}[1-\theta(\xi, 0)], \phi(\xi, 0)=1, \\
f^{\prime}(\xi, \infty)=0, \quad g(\xi, \infty)=0, \theta(\xi, \infty)=0, \phi(\xi, \infty)=0 .
\end{array}
$$

where $B i=h_{f} L /\left(k_{f} G r^{1 / 4}\right)$ is the Biot number.

$$
\text { Non-dimensional shear stress } C_{f}=\frac{2}{\rho U_{*}^{2}}\left[(\mu+\kappa) \frac{\partial u}{\partial y}+\kappa \omega\right]_{y=0},
$$
the wall couple stress $M_{w}=\frac{\gamma}{\rho U_{*}^{2} L}\left[\frac{\partial \omega}{\partial y}\right]_{y=0}$, the Nusselt number $N u_{x}=-\frac{x}{\left(T_{f}-T_{\infty}\right)}\left[\frac{\partial T}{\partial y}\right]_{y=0}$ and the Sherwood number $S h_{x}=-\frac{x}{\left(C_{w}-C_{\infty}\right)}\left[\frac{\partial C}{\partial y}\right]_{y=0}$ are given by

$$
\begin{array}{r}
C_{f} G r_{x}^{\frac{1}{4}}=\left(\frac{2}{1-N}\right) f^{\prime \prime}(\xi, 0), M_{w} G r_{x}^{\frac{1}{2}}=\left(\frac{\lambda}{\mathcal{J}}\right) \xi^{\frac{1}{2}} g^{\prime}(\xi, 0), \\
N u_{x} G r_{x}^{\frac{-1}{4}}=-\theta^{\prime}(\xi, 0), S h_{x} G r_{x}^{\frac{-1}{4}}=-\phi^{\prime}(\xi, 0) .
\end{array}
$$

where $U_{*}$ and $G r_{x}=\left[g^{*} \beta_{0}\left(T_{f}-T_{\infty}\right) x^{3}\right] / \nu^{2}$ are the characteristic velocity and the local thermal Grashof number, respectively.

\section{SOLUTION OF THE PROBLEM}

We now obtain approximate solutions to Eqs. (9) - (12) together with the boundary conditions (13) in two steps: (i) First, we use, the local nonsimilarity procedure to convert the set of partial differential equations (9)-(12) along with the boundary conditions (13) into ordinary differential equations system, (ii) Next, the resulting set of nonlinear ordinary differential equations is evaluated using a Successive Linearisation Method (SLM), it utilizes first the successive linearization and then the Chebyshev spectral collocation scheme. According to local similarity and nonsimilarity procedure of Sparrow and Yu (1971), the set of non-similar equations considered here are first transformed to a set of ordinary nonlinear differential equations by introducing new unknown functions of $\xi$ derivatives. The preliminary approximate solution can be found from local similarity equations for a particular case of $\xi<<1$ and the terms containing $\xi \frac{\partial}{\partial \xi}$ are supposed to be very small. So that we can neglect terms containing the $\xi$ derivatives in equations (9)-(13). Thus the first level truncation or local similarity equations are

$$
\begin{aligned}
& \frac{1}{\varepsilon}\left(\frac{1}{1-N}\right) f^{\prime \prime \prime}+\frac{3}{4 \varepsilon^{2}} f f^{\prime \prime}-\frac{1}{2 \varepsilon^{2}} f^{\prime 2}+\left(\frac{N}{1-N}\right) g^{\prime}-\frac{F s}{D a} \xi f^{\prime 2} \\
& -\frac{1}{D a G r^{\frac{1}{2}}} \xi^{\frac{1}{2}} f^{\prime}+\left[\theta\left(1+\alpha_{1} \theta\right)+\mathcal{B} \phi\left(1+\alpha_{2} \phi\right)\right] \cos \Omega=0
\end{aligned}
$$

$$
\begin{gathered}
\lambda g^{\prime \prime}-\frac{1}{4 \varepsilon} g f^{\prime}+\frac{3}{4 \varepsilon} g^{\prime} f-\left(\frac{N}{1-N}\right) \mathcal{J} \xi^{\frac{1}{2}}\left(\frac{1}{\varepsilon} f^{\prime \prime}+2 g\right)=0 \\
\frac{1}{\operatorname{Pr}} \theta^{\prime \prime}+\frac{3}{4} f \theta^{\prime}=0 \\
\frac{1}{S c} \phi^{\prime \prime}+\frac{3}{4} f \phi^{\prime}=0
\end{gathered}
$$

The associated boundary conditions are

$$
\begin{gathered}
f^{\prime}(\xi, 0)=f(\xi, 0)=g(\xi, 0)=0, \theta^{\prime}(\xi, 0)+B i \xi^{\frac{1}{4}}[1-\theta(\xi, 0)] \\
\phi(\xi, 0)=1, f^{\prime}(\xi, \infty)=g(\xi, \infty)=\theta(\xi, \infty)=\phi(\xi, \infty)=0 .
\end{gathered}
$$

The local non-similarity ordinary nonlinear differential equations in the second level truncation is discovered by introducing new variables to recall the omitted expressions from the first level truncation i.e. take $U=\frac{\partial f}{\partial \xi}, V=\frac{\partial g}{\partial \xi}, H=\frac{\partial \theta}{\partial \xi}, K=\frac{\partial \phi}{\partial \xi}$. Thus the second level truncation is

$$
\begin{aligned}
\frac{1}{\varepsilon}\left(\frac{1}{1-N}\right) f^{\prime \prime \prime}+\frac{3}{4 \varepsilon^{2}} f f^{\prime \prime}-\frac{1}{2 \varepsilon^{2}} f^{\prime 2}+ & \left(\frac{N}{1-N}\right) g^{\prime}-\frac{F s}{D a} \xi f^{\prime 2} \\
-\frac{1}{D a G r^{\frac{1}{2}}} \xi^{\frac{1}{2}} f^{\prime}+\left[\theta\left(1+\alpha_{1} \theta\right)\right. & \left.+\mathcal{B} \phi\left(1+\alpha_{2} \phi\right)\right] \cos \Omega \\
& =\frac{\xi}{\varepsilon^{2}}\left(f^{\prime} U^{\prime}-f^{\prime \prime} U\right) \\
\lambda g^{\prime \prime}+\frac{3}{4 \varepsilon} f g^{\prime}-\frac{1}{4 \varepsilon} f^{\prime} g-\left(\frac{N}{1-N}\right) & =\frac{\xi}{\varepsilon} \xi^{\frac{1}{2}}\left(2 g+\frac{1}{\varepsilon} f^{\prime \prime}\right) \\
\frac{1}{\operatorname{Pr}} \theta^{\prime \prime}+\frac{3}{4} f \theta^{\prime} & =\xi\left(f^{\prime} H-g^{\prime} U\right) \\
\frac{1}{S c} \phi^{\prime \prime}+\frac{3}{4} f \phi^{\prime} & =\xi\left(f^{\prime} K-\phi^{\prime} U\right)
\end{aligned}
$$

The corresponding boundary conditions are

$$
\begin{array}{r}
f(\xi, 0)+\frac{4}{3} \xi U(\xi, 0)=f^{\prime}(\xi, 0)=g(\xi, 0)=0, \theta^{\prime}(\xi, 0) \\
-B i \xi^{\frac{1}{4}}[\theta(\xi, 0)-1]=0, \phi(\xi, 0)=1, f^{\prime}(\xi, \infty)=g(\xi, \infty) \\
=\theta(\xi, \infty)=\phi(\xi, \infty)=0 .
\end{array}
$$

The two level local non-similarity technique is accomplished with a third level of truncation, for this we differentiate equations (20)-(24) with respect to $\xi$ and omit the partial derivatives of $U, V, H, K$.Then the resultant equations are

$$
\begin{gathered}
\frac{1}{\varepsilon}\left(\frac{1}{1-N}\right) U^{\prime \prime \prime}+\frac{7}{4 \varepsilon^{2}} U f^{\prime \prime}+\frac{3}{4 \varepsilon^{2}} f U^{\prime \prime}+\left(\frac{N}{1-N}\right) V^{\prime}-\frac{\xi^{\frac{-1}{2}}}{2 D a G r^{\frac{1}{2}}} f^{\prime} \\
-\frac{\xi^{\frac{1}{2}}}{D a \mathrm{Gr} \frac{1}{2}} U^{\prime}-\frac{F s}{D a} f^{\prime 2}+\left[H\left(1+2 \alpha_{1} \theta\right)+\mathcal{B} K\left(1+2 \alpha_{2} \phi\right)\right] \cos \Omega \\
-2\left[\frac{F s}{D a} \xi+\frac{1}{\varepsilon^{2}}\right] f^{\prime} U^{\prime}=\frac{\xi}{\varepsilon^{2}}\left(U^{\prime 2}-U^{\prime \prime} U\right) \\
\lambda V^{\prime \prime}+\frac{7}{4 \varepsilon} U g^{\prime}+\frac{3}{4 \varepsilon} f V^{\prime}-\frac{1}{4 \varepsilon} U^{\prime} g-\frac{1}{2}\left(\frac{N}{1-N}\right) \mathcal{J} \xi^{\frac{-1}{2}}\left(2 g+\frac{1}{\varepsilon} f^{\prime \prime}\right) \\
-\frac{5}{4 \varepsilon} V f^{\prime}-\left(\frac{N}{1-N}\right) \mathcal{J} \xi^{\frac{1}{2}}\left(2 V+\frac{1}{\varepsilon} U^{\prime \prime}\right)=\frac{\xi}{\varepsilon}\left(V U^{\prime}-V^{\prime} U\right) \\
\frac{1}{P r} H^{\prime \prime}+\frac{7}{4} U \theta^{\prime}+\frac{3}{4} f H^{\prime}-f^{\prime} H=\xi\left(H U^{\prime}-H^{\prime} U\right) \\
\frac{1}{S c} K^{\prime \prime}+\frac{7}{4} U \phi^{\prime}+\frac{3}{4} f K^{\prime}-f^{\prime} K=\xi\left(K U^{\prime}-K^{\prime} U\right)
\end{gathered}
$$


Table 2 A comparison of $f^{\prime \prime}(\xi, 0),-g^{\prime}(\xi, 0),-\theta^{\prime}(\xi, 0),-\phi^{\prime}(\xi, 0)$ using the SLM and Shooting method for different values of $N$ with $\mathcal{B}=0.5$, $\varepsilon=0.3, \alpha_{1}=0, \alpha_{2}=0, D a \rightarrow \infty, \lambda=5.0, J=5.0, B i \rightarrow \infty, \Omega=0, \operatorname{Pr}=0.7$ and $S c=0.22$.

\begin{tabular}{|c|cccc|cccc|}
\hline & \multicolumn{3}{|c|}{ SLM } & \multicolumn{3}{c|}{ Shooting } & Method \\
\hline$N$ & $f^{\prime \prime}(0.2,0)$ & $-g^{\prime}(0.2,0)$ & $-\theta^{\prime}(0.2,0)$ & $-\phi^{\prime}(0.2,0)$ & $f^{\prime \prime}(0.2,0)$ & $-g^{\prime}(0.2,0)$ & $-\theta^{\prime}(0.2,0)$ & $-\phi^{\prime}(0.2,0)$ \\
\hline 0.1 & 0.44697 & 0.028502 & 0.25942 & 0.13309 & 0.44697 & 0.028502 & 0.25942 & 0.13309 \\
0.3 & 0.38280 & 0.114050 & 0.25303 & 0.13114 & 0.38280 & 0.114050 & 0.25303 & 0.13114 \\
0.6 & 0.26376 & 0.295950 & 0.23859 & 0.12661 & 0.26376 & 0.295950 & 0.23859 & 0.12661 \\
0.9 & 0.09660 & 0.491030 & 0.20265 & 0.11428 & 0.09660 & 0.491030 & 0.20265 & 0.11428 \\
\hline
\end{tabular}

The corresponding boundary conditions are

$$
\begin{array}{r}
U^{\prime}(\xi, 0)=U(\xi, 0)=V(\xi, 0)=0 ; H^{\prime}(\xi, 0)-B i \xi^{\frac{1}{4}} H(\xi, 0) \\
+\frac{1}{4} B i \xi^{\frac{-3}{4}}[1-\theta(\xi, 0)]=0 ; K(\xi, 0)=U^{\prime}(\xi, \infty)=V(\xi, \infty) \\
=H(\xi, \infty)=K(\xi, \infty)=0 .
\end{array}
$$

The coupled nonlinear differential Eqs. (20) - (23) and Eqs. (25) - (28) along with the boundary conditions (24) and (29) are evaluated using one of the non-perturbation method named as Successive Linearization Method(for more details see, Makukula et al., 2010; Awad et al., 2011; Khidir et al., 2015 ). Using the successive linearization technique, the nonlinear boundary layer equations will reduce to a system of linear differential equations. For this, let us consider an independent vector $\mathbb{Q}(\eta)=[f(\eta), g(\eta), \theta(\eta), \phi(\eta), U(\eta), V(\eta), H(\eta), K(\eta)]$ and assume that it can be represented as

$$
\mathbb{Q}(\eta)=\mathbb{Q}_{n}(\eta)+\sum_{m=0}^{n-1} \mathbb{Q}_{m}(\eta)
$$

where $\mathbb{Q}_{n}(\eta), n=1,2,3 \ldots$ are unknown vectors those are determined by recursively evaluating the linearised version of the governing equation and presuming that $\mathbb{Q}_{m}(\eta),(0 \leq m \leq n-1)$ are expected from antecedent iterations. The initial guess $\mathbb{Q}_{0}(\eta)$ is selected so that it satisfy the boundary conditions (24) and (29). By imposing Eq.(30) in Eqs.(20)(28) and considering only linear terms, we get the linearised equations to be evaluated are

$$
\begin{array}{r}
\tilde{p}_{1, n-1} f_{n}^{\prime \prime \prime}+\tilde{p}_{2, n-1} f_{n}^{\prime \prime}+\tilde{p}_{3, n-1} f_{n}^{\prime}+\tilde{p}_{4, n-1} f_{n}+\tilde{p}_{5, n-1} g_{n}^{\prime} \\
+\tilde{p}_{6, n-1} \theta_{n}+\tilde{p}_{7, n-1} \phi_{n}+\tilde{p}_{8, n-1} U_{n}^{\prime}+\tilde{p}_{9, n-1} U_{n}=\tilde{r}_{1, n-1} \\
\tilde{q}_{1, n-1} f_{n}^{\prime \prime}+\tilde{q}_{2, n-1} f_{n}^{\prime}+\tilde{q}_{3, n-1} f_{n}+\tilde{q}_{4, n-1} g_{n}^{\prime \prime}+\tilde{q}_{5, n-1} g_{n}^{\prime} \\
+\tilde{q}_{6, n-1} g_{n}+\tilde{q}_{7, n-1} U_{n}+\tilde{q}_{8, n-1} V_{n}=\tilde{r}_{2, n-1} \\
\tilde{s}_{1, n-1} f_{n}^{\prime}+\tilde{s}_{2, n-1} f_{n}+\tilde{s}_{3, n-1} \theta_{n}^{\prime \prime}+\tilde{s}_{4, n-1} \theta_{n}^{\prime}+\tilde{s}_{5, n-1} U_{n} \\
+\tilde{s}_{6, n-1} H_{n}=\tilde{r}_{3, n-1} \\
\tilde{t}_{1, n-1} f_{n}^{\prime}+\tilde{t}_{2, n-1} f_{n}+\tilde{t}_{3, n-1} \phi_{n}^{\prime \prime}+\tilde{t}_{4, n-1} \phi_{n}^{\prime}+\tilde{t}_{5, n-1} U_{n} \\
+\tilde{t}_{6, n-1} K_{n}=\tilde{r}_{4, n-1} \\
\tilde{a}_{1, n-1} f_{n}^{\prime \prime}+\tilde{a}_{2, n-1} f_{n}^{\prime}+\tilde{a}_{3, n-1} f_{n}+\tilde{a}_{4, n-1} \theta_{n}+\tilde{a}_{5, n-1} \phi_{n} \\
+\tilde{a}_{6, n-1} U_{n}^{\prime \prime \prime}+\tilde{a}_{7, n-1} U_{n}^{\prime \prime}+\tilde{a}_{8, n-1} U_{n}^{\prime}+\tilde{a}_{9, n-1} U_{n} \\
+\tilde{a}_{10, n-1} V_{n}^{\prime}+\tilde{a}_{11, n-1} H_{n}+\tilde{a}_{12, n-1} K_{n}=\tilde{r}_{5, n-1} \\
\tilde{b}_{1, n-1} f_{n}^{\prime \prime}+\tilde{b}_{2, n-1} f_{n}^{\prime}+\tilde{b}_{3, n-1} f_{n}+\tilde{b}_{4, n-1} g_{n}^{\prime}+\tilde{b}_{5, n-1} g_{n}+ \\
\tilde{b}_{6, n-1} U_{n}^{\prime \prime}+\tilde{b}_{7, n-1} U_{n}^{\prime}+\tilde{b}_{8, n-1} U_{n}+\tilde{b}_{9, n-1} V_{n}^{\prime \prime}+\tilde{b}_{10, n-1} V_{n}^{\prime} \\
\quad+\tilde{b}_{11, n-1} V_{n}=\tilde{r}_{6, n-1} \\
\tilde{c}_{1, n-1} f_{n}^{\prime}+\tilde{c}_{2, n-1} f_{n}+\tilde{c}_{3, n-1} \theta_{n}^{\prime}+\tilde{c}_{4, n-1} U_{n}^{\prime}+\tilde{c}_{5, n-1} U_{n} \\
+\tilde{c}_{6, n-1} H_{n}^{\prime \prime}+\tilde{c}_{7, n-1} H_{n}^{\prime}+\tilde{c}_{8, n-1} H_{n}=\tilde{r}_{7, n-1} \\
\tilde{d}_{1, n-1} f_{n}^{\prime}+\tilde{d}_{2, n-1} f_{n}+\tilde{d}_{3, n-1} \phi_{n}^{\prime}+\tilde{d}_{4, n-1} U_{n}^{\prime}+\tilde{d}_{5, n-1} U_{n} \\
+\tilde{d}_{6, n-1} K_{n}^{\prime \prime}+\tilde{d}_{7, n-1} K_{n}^{\prime}+\tilde{d}_{8, n-1} K_{n}=\tilde{r}_{8, n-1}
\end{array}
$$

The boundary conditions reduce to

$$
\begin{array}{r}
f_{n}(0)=f_{n}^{\prime}(0)=f_{n}^{\prime}(\infty)=0, g_{n}(0)=g_{n}(\infty)=0, \phi_{n}(\infty)=0, \phi_{n}(0)=\phi_{n}(\infty)=0, \\
\theta_{n}^{\prime}(0)-B i \xi^{\frac{1}{4}} \theta_{n}(0)=0, \theta_{n}(\infty)=0, \\
U_{n}(0)=U_{n}^{\prime}(0)=U_{n}^{\prime}(\infty)=0, V_{n}(0)=V_{n}\left(\infty \xi^{\frac{1}{4}} H_{n}(0)-\frac{1}{4} B i \xi^{\frac{-3}{4}} \theta_{n}(0)=0, H_{n}(\infty)=0,\right. \\
K_{n}(0)=K_{n}(\infty)=0 .
\end{array}
$$

Here the coefficient parameters $\tilde{p}_{i, n-1}, \tilde{q}_{i, n-1}, \tilde{s}_{i, n-1}, \tilde{t}_{i, n-1}, \tilde{a}_{i, n-1,}$, $\tilde{b}_{i, n-1}, \tilde{c}_{i, n-1}, \tilde{d}_{i, n-1}$ and $\tilde{r}_{i, n-1}$ depend on the initial guesses $\mathbb{Q}_{0}(\eta)$ and on their derivatives.

We solve linearised Eqs. (31) - (38) by an established procedure, namely Chebyshev collocation method( Canuto et al., 2006). In the context of numerical implication, the original region $[0, \infty)$ is truncated to $[0, L]$ for large value of $L$, and further the truncated region $[0, L]$ is transformed into $[-1,1]$ using the following mapping

$$
\frac{\eta}{L}=\frac{\tau+1}{2}, \quad-1 \leq \tau \leq 1
$$

In this procedure, the Chebyshev polynomials $T_{w}(\tau)=\cos \left[w \cos ^{-1} \tau\right]$ are used to approximate the unknown functions $\mathbb{Q}_{n}(\eta)$ and these polynomials are collocated at $K+1$ Gauss-Lobatto points in the interval $[-1,1]$ and those are defined as

$$
\tau_{m}=\cos \frac{\pi m}{K}, \quad m=0,1, \ldots, K
$$

The unknown function $\mathbb{Q}_{n}(\eta)$ is imprecise at the collocation points by

$$
\begin{array}{r}
\mathbb{Q}_{n}(\tau)=\sum_{i=0}^{K} \mathbb{Q}_{n}\left(\tau_{i}\right) T_{i}\left(\tau_{m}\right) \text { and } \frac{d^{\mathbb{Z}}}{d \eta^{\mathbb{Z}}} \mathbb{Q}_{n}(\tau)=\sum_{i=0}^{K} \mathbf{D}_{i m}^{\mathbb{Z}} \mathbb{Q}_{n}\left(\tau_{i}\right), \\
m=0,1, \ldots K
\end{array}
$$

where $\mathcal{D}$ is the Chebyshev spectral derivative matrix such that $\mathbf{D}=$ $(2 / L) \mathcal{D}$ and $\mathbb{Z}$ is the order of differentiation. After employing Eqs.(40) (42) into linearized form of equations (31)- (38), the resultant solution is

$$
\tilde{\mathbf{Y}}_{n}=\tilde{\mathbf{B}}_{n-1}^{-1} \tilde{\mathbf{R}}_{n-1}
$$

In Eq.(43), $\tilde{\mathbf{B}}_{n-1}$ is a square matrix of order $(8 K+8) \times(8 K+8)$ and $\tilde{\mathbf{Y}}_{n}, \tilde{\mathbf{R}}_{n-1}$ are column matrix of order $(8 K+1) \times 1$, which are defined by

$$
\tilde{\mathbf{B}}_{n-1}=\left[\tilde{\mathbf{B}}_{p q}\right], p, q=1,2, \ldots 8, \tilde{\mathbf{X}}_{n}=
$$$$
\left[\begin{array}{llllllll}
\tilde{\mathbb{F}}_{n} & \tilde{\mathbb{G}}_{n} & \tilde{\Theta}_{n} & \tilde{\Phi}_{n} & \tilde{\mathbb{U}}_{n} & \tilde{\mathbb{V}}_{n} & \tilde{\mathbb{H}}_{n} & \tilde{\mathbb{K}}_{n}
\end{array}\right]^{T}, \tilde{\mathbf{R}}_{n-1}=
$$ 


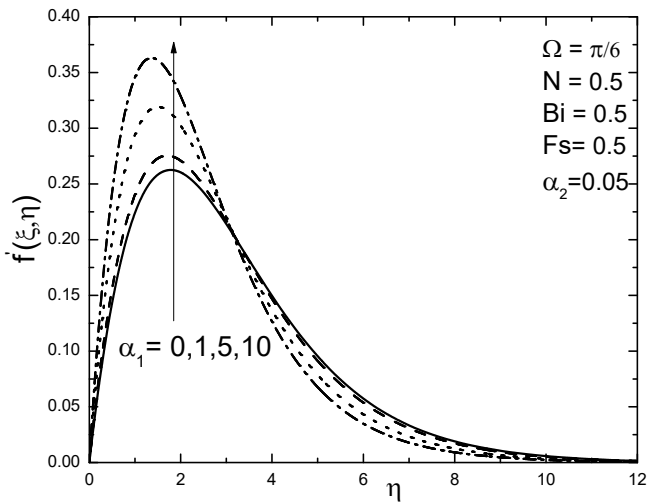

(a)

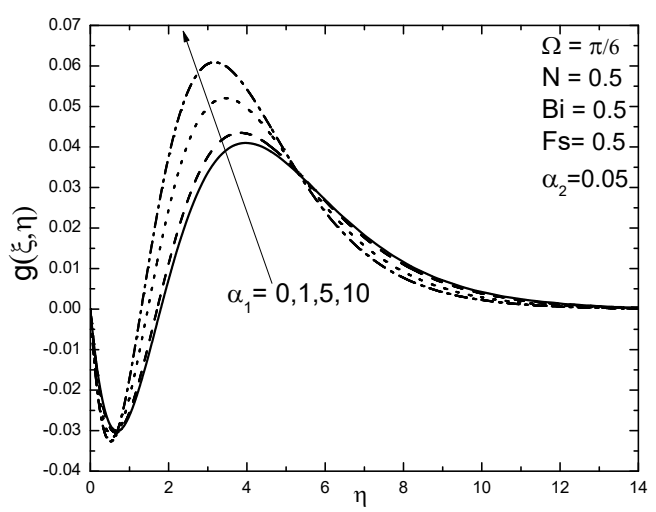

(b)

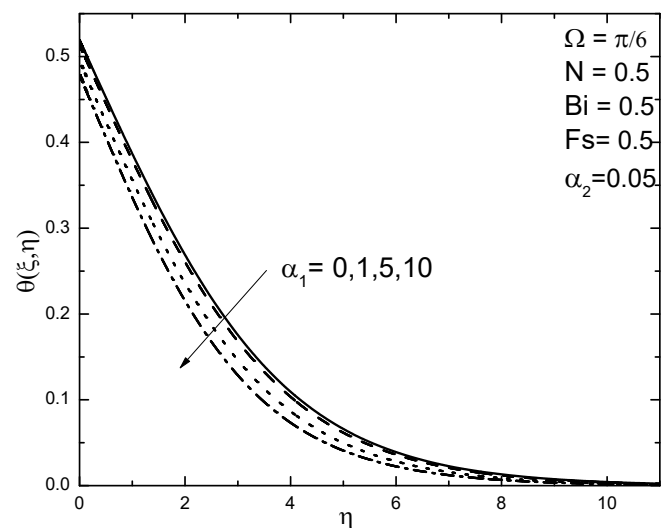

(c)

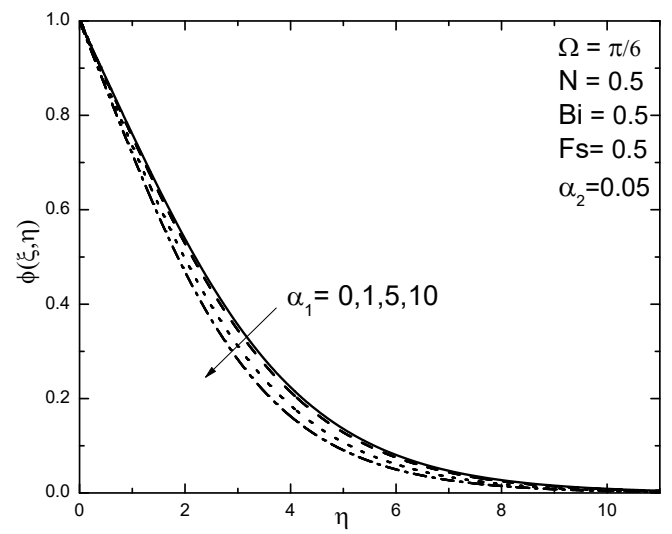

(d)

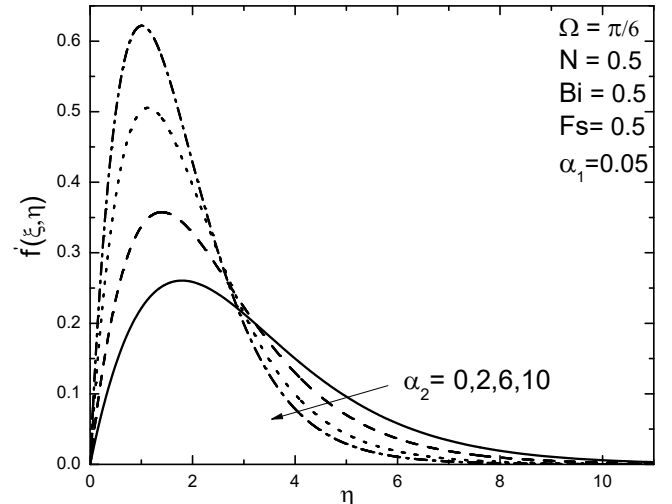

(a)

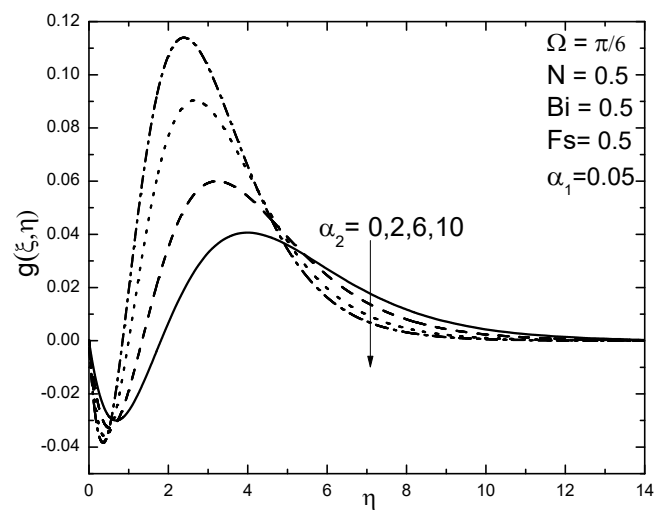

(b)

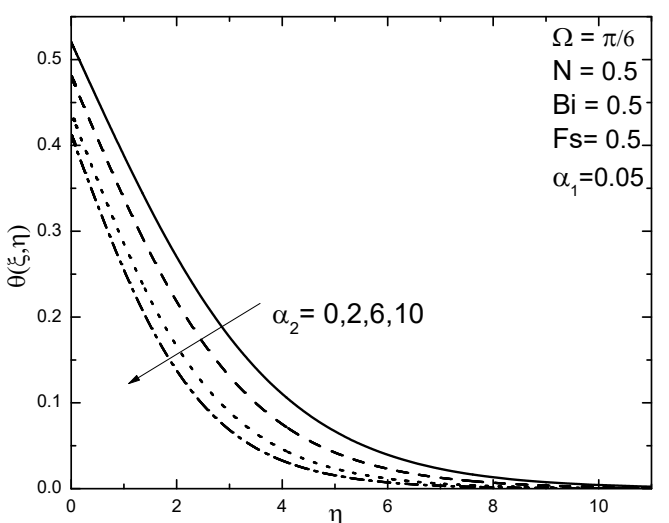

(c)

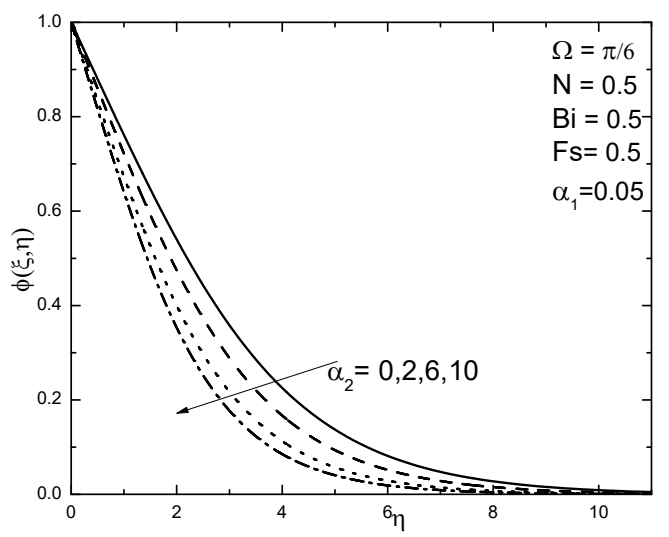

(d)
Fig. 2 Effect of $\alpha_{1}$ on the (a) velocity, (b) microrotation, (c) temperature and $(d)$ concentration.
Fig. 3 Effect of $\alpha_{2}$ on the (a) velocity, (b) microrotation, (c) temperature and $(d)$ concentration. 


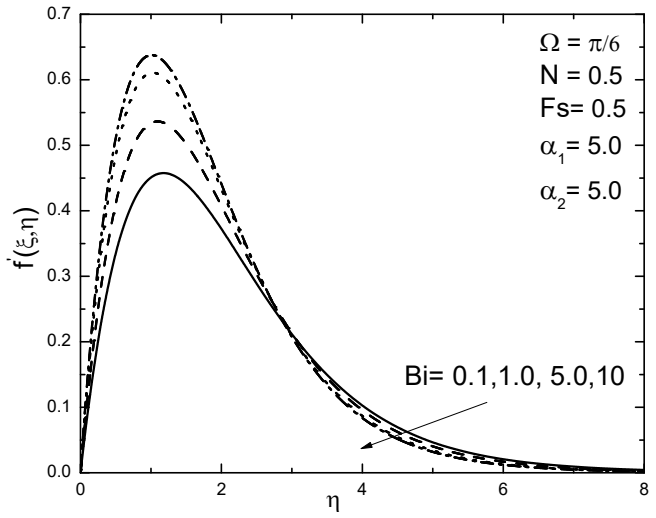

(a)

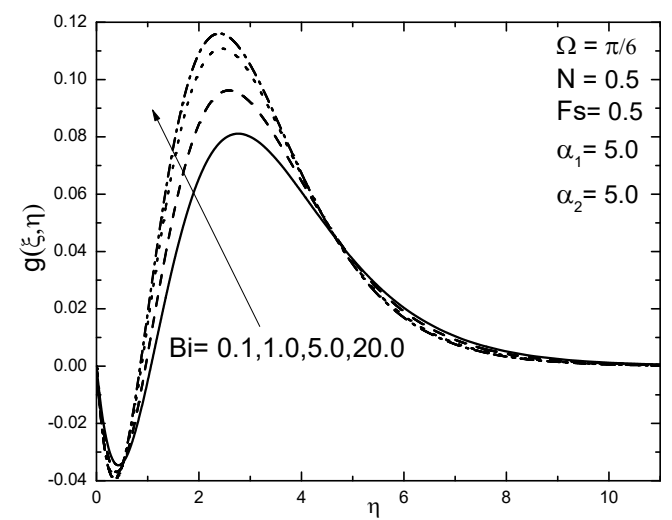

(b)

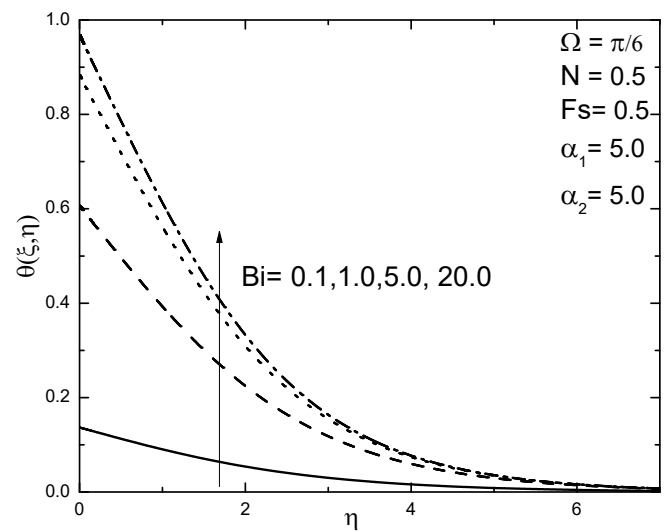

(c)

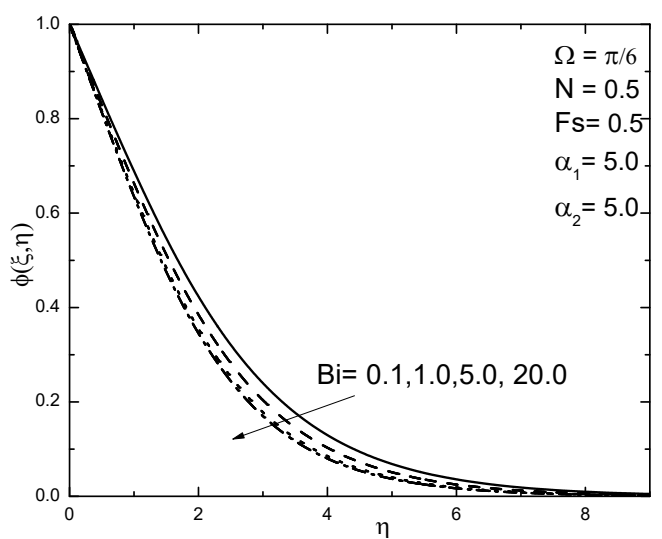

(d)

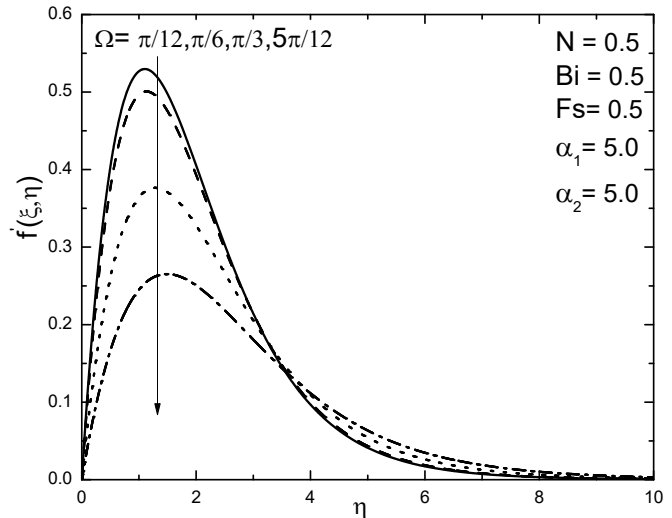

(a)

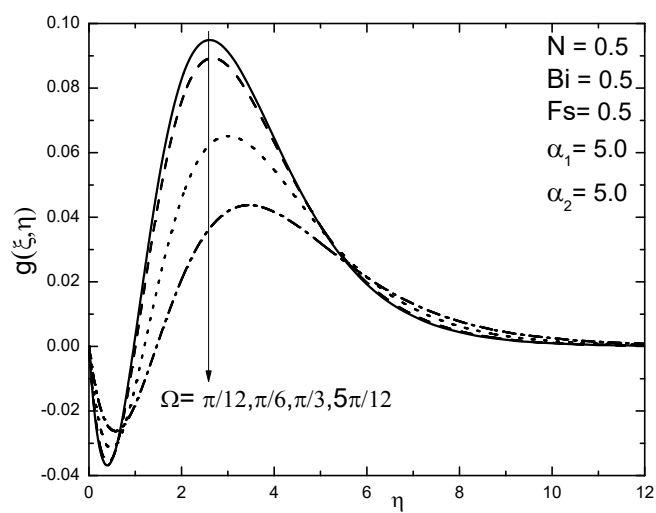

(b)

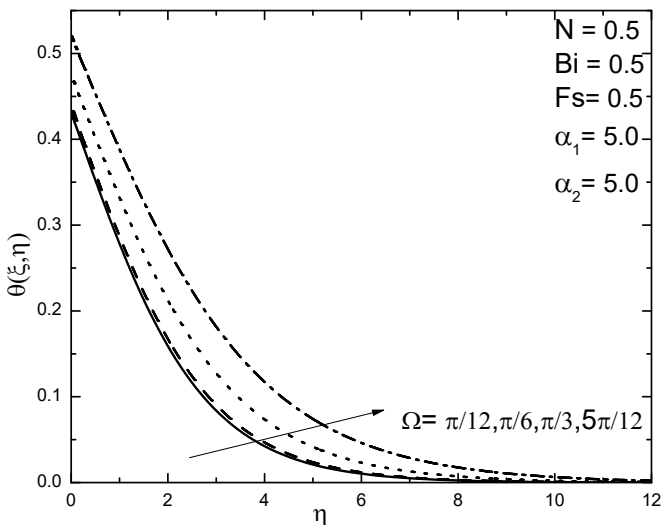

(c)

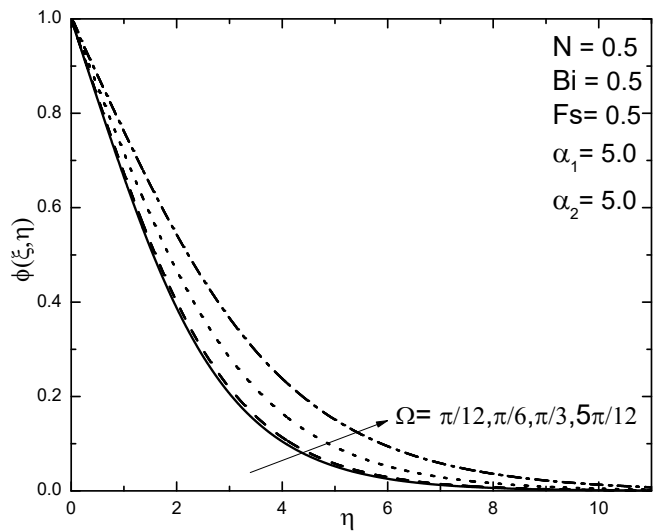

(d)
Fig. 4 Effect of Bi on the (a) velocity, (b) microrotation, (c) temperature and $(d)$ concentration.
Fig. 5 Effect of $\Omega$ on the (a) velocity, (b) microrotation, (c) temperature 6 


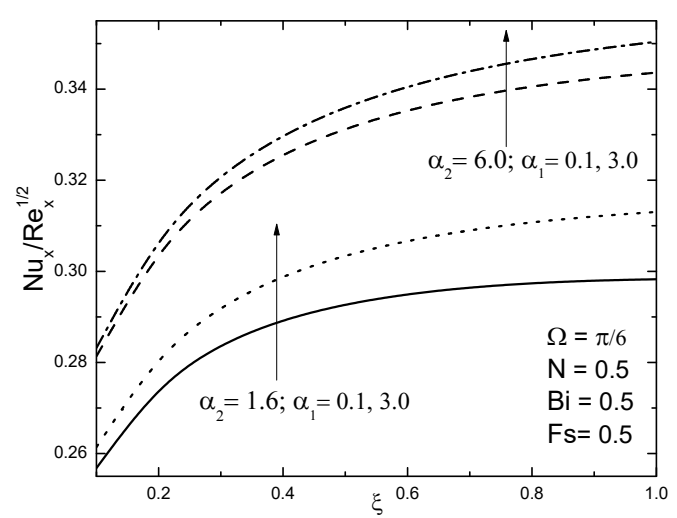

(a)

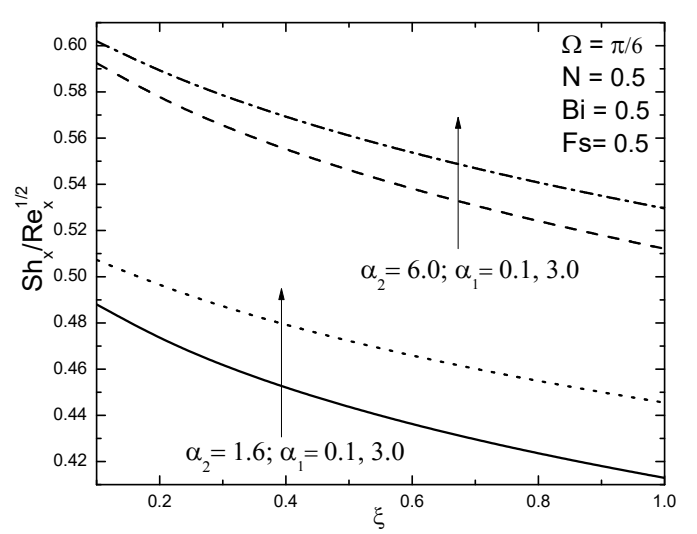

(b)

Fig. 6 Effect of $\alpha_{1}$ and $\alpha_{2}$ on the (a) Nusselt (b) Sherwood number's.

\section{RESULTS AND DISCUSSIONS}

It is noticed that the present problem reduces to free convection heat transfer over an isothermal vertical plate in a viscous fluid in the absence of coupling number, buoyancy ratio, nonlinear convection parameters with $\epsilon=1, D a \rightarrow \infty, \lambda=0, \xi=1, B i \rightarrow \infty$ and $\Omega=0$. In order to assess the accuracy and validity of the present investigation, the results of the local similarity equations (15)-(18) have been compared with the regular fluid which is reported in Tab. (4.2) of Bejan (2013) as exhibited in Tab. 1. Also, the comparison between the SLM and shooting method has been made for certain values of the physical parameters as shown in the Tab. 2. It shows an excellent agreement with existing results. As per the restrictions of thermodynamics on the material parameters mentioned by Eringen (1966), the numerical computations are carried out by following default parameter values: $\mathcal{J}=5.0, \lambda=5.0$, $\mathcal{B}=1.0, \operatorname{Pr}=0.7, S c=0.22, G r=10, \varepsilon=0.5, D a=0.1$ and $\xi=0.1$ and these values are continued throughout the evaluation, unless otherwise designated. Figures 2(a)-5(d) illustrate the influence of various parameters on the fluid for the velocity, microrotation, temperature and concentration profiles. The effects of nonlinear temperature, nonlinear concentration, non-Darcy parameter, micropolar parameter, Biot number and inclination of angle on heat and mass characteristics of the fluid have been discussed and reported in Figs. 6(a) -8(b). The effect of the nonlinear density-temperature (NDT) parameter $\alpha_{1}$ on the microrotation, velocity, temperature and the concentration profiles are displayed in Figs. 2(a)-2(d). As NDT parameter $\alpha_{1}$ increases, the direction of fluid velocity is changed from increasing to decreasing nearer to the free stream value as shown in Fig. 2(a). Physically, $\alpha_{1}>0$ implies that $T_{f}>T_{\infty}$; hence, the surface of the wall will induce some amount of heat to the fluid flow region. Similarly, there is a possibility for heat transformation of fluid flow to the wall in the case of $\alpha_{1}<0$. It is clearly noticed from Fig. 2(b) that the effect of the NDT parameter $\alpha_{1}$ on the microrotation is notable in every part of the boundary layer, but more significant away from the plate and also the rise of $\alpha_{1}$ changes the sign of microrotation. The magnitude of the temperature and concentration are more in the absence of NDT parameter $\alpha_{1}$ in comparison with the presence of NDT parameter $\alpha_{1}$ as shown in Figs. 2(c) - 2(d).

Figures 3(a) to 3(d) depict the influence of the nonlinear densityconcentration (NDC) parameter $\alpha_{2}$ for a fixed value of $\alpha_{1}=0.05$ on the behavior of velocity, microrotation, temperature and concentration. Usually, the motion of the fluid flow increases sequentially away from the plate so that it reaches an eminent position within the boundary layer and then drops to fulfill the free stream values as given in Fig. 3(a). However, the rise of NDC parameter $\alpha_{2}$ changes the sign of microrotation within the boundary layer from negative to positive and also the trend of microrotation is modified from enhancing to diminish nearer to the free stream value $\eta=5$, as shown if Fig. 3(b). The concentration and temperature boundary layer thicknesses decrease with the rise of $\alpha_{2}$ and the same effect is displayed in Figs. 3(c) and 3(d). In the case of thermal and concentration distributions, present results correlate with the findings of Partha (2010). This is due to the enhancement of thermal and solutal gradients by nonlinear terms in the momentum equation. By the experience of these two NDT and NDC parameters, we conclude that the authority of NDC parameter $\alpha_{2}$ is more prominent compared with that of NDT parameter $\alpha_{1}$.

The profiles of fluid flow for different values of conductive-convective parameter $B i$ has been displayed in Figs. 4(a) - 4(d). It is noteworthy, from Figs.4(a) - 4(b) that as the Biot number increases, the velocity of flow field attains peak state in the neighborhood of the plate due to the reduction in the thermal resistance of the inclined plate. Also, the direction of the velocity and the microrotation changes from increasing to decreasing values within the boundary layer. As seen in Aziz (2009), Fig. 4(c) signifies the impact Biot number of on the temperature distribution and serves as a dual result (i.e., for isothermal and non-isothermal condition). Since the specified convective boundary condition is changing into wall condition, when the Biot number tends to infinity and it is proved, which is displayed in Fig. 4(c). As $B i$ increases from thermally thin case $B i<0.1$ to thermally thick case $B i>0.1$, the temperature distribution is increased on the surface of the plate. The effect of the Biot number on the concentration profile is displayed by Fig. 4(d) and it depicts that the concentration profile decreases within the boundary layer with the increasing value of the Biot number. The specified temperature and concentration profiles are subjectively equal with those of Makinde and Aziz (2011) who investigated the results for a nanofluid over a stretching sheet.

The influence of inclination of angle $(\Omega)$ on the tangential velocity profile is displayed in Fig. 5(a). Due to the reduction in the thermal and concentration buoyancy effect in Eq.(2), an increase in the inclination of angle leads to reduce the velocity distribution within the boundary layer region. From Fig.5(b), one can notice that the microrotation is increasing near the plate and it is showing a reverse trend far away from the plate within the boundary layer when the position of a flat plate is changed from vertical to horizontal. Similar to the results of Chamkha et al. (2002) and Chen (2004), the thickness of temperature and concentration boundary layers enhances with rising values of inclination of angle. Since the displacement of the plate from vertical position to horizontal induces reduction in buoyancy force, the same effect projected in Figs. 5(c) and 5(d). Also, one can observe that the maximum buoyancy force for the same temperature and concentration difference occurs for $\Omega=0$ (vertical plate) and this is noticed in Figs. 5(a) - 5(d).

The effects of the nonlinear density-temperature (NDT) and concentration (NDC) parameters $\alpha_{1}$ and $\alpha_{2}$ respectively on the mass and heat transfer rates against streamwise coordinate $\xi$ shown in Figs. 6(a) 6(b). As expected, both mass and heat transfer rates are increasing with $\alpha_{1}$ when $\alpha_{2}$ is fixed. The effect of $\alpha_{2}$ on mass and heat transfer rates is 


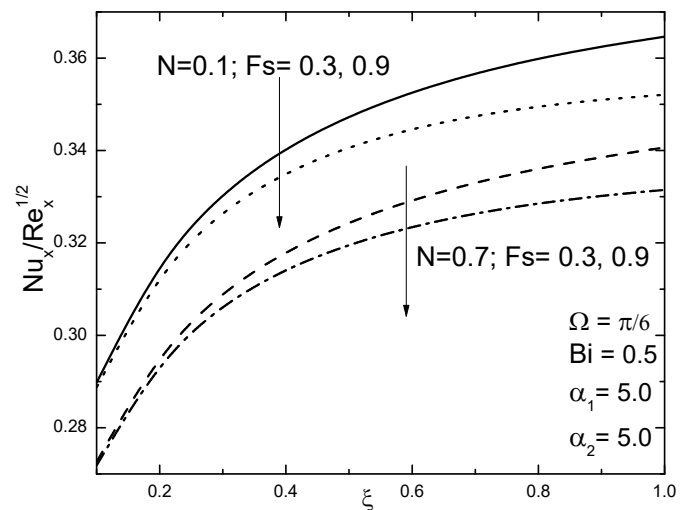

(a)

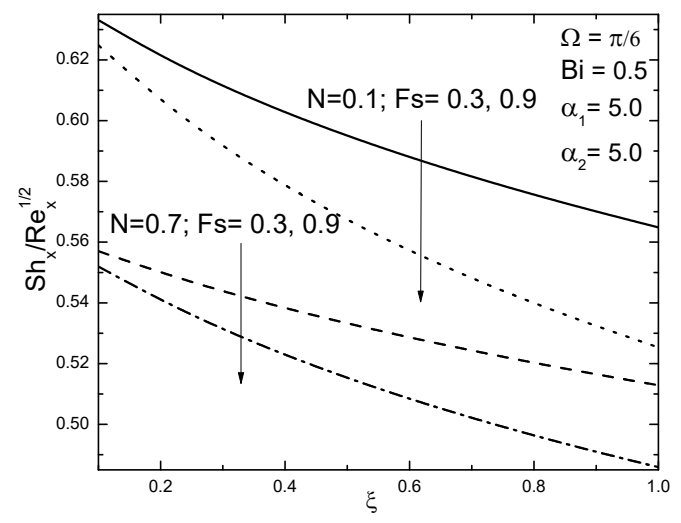

(b)

Fig. 7 Effect of Fs and $N$ on the (a) Nusselt (b) Sherwood number's.

showing the same behavior like that of $\alpha_{1}$. But, along streamwise coordinate $\xi$ the nature of mass transfer rate is contrary to the heat transfer rate. The effect of varying the Forchheimer number $F s$ and Coupling number $N$ on mass and heat transfer rates are presented in Figs. 7(a) - 7(b) The results point out that as Fs increases, the mass and heat transfer rates decrease for a fixed value of coupling number. The rise of coupling number reduces the mass and heat transfer rates. Figs. 8(a) - 8(b) demonstrate the influence of Biot number $B i$ and the variation of the inclination of angle $\Omega$ on the mass and heat transfer rates. It is found that the mass and heat transfer rates diminish when the inclined plate rotating from vertical to horizontal, and it is easily perceived from the fact that there is a reduction in the buoyancy force by a function of $\cos \Omega$ as the inclination of angle increases. With the enhancement of Biot number, there is a considerable increment in mass and heat transfer rates. The modification in the Biot number (i.e from thermally thin case to thick case) ceases the rate of heat conduction inside the inclined plate drastically as compared to thermal convection away from its surface. These results are confined to the work of Bég et al. (2014). One can notice that the effect of angle of inclination is more on mass transfer rate $S h$ as compared with that of heat transfer rate $N u$.

The proportional quantities of skin friction and the gradient of microrotation (wall couple stress) are computed for the enhanced values of $N, F s, \alpha_{1}, \alpha_{2}, \Omega, B i$ and the results are presented in Tab. 3. It is observed that an enhancement in the Biot number causes an increase in the couple stress and skin friction, whereas with the increasing of the angle of inclination they show the opposite trend. An increase in the coupling number tends to reduce the drag coefficient and the opposite change is noticed in wall couple stress. The rate of wall couple stress and skin friction are enhanced by nonlinear density-temperature and concentration parameters, in which the influence of NDT parameter is nominal

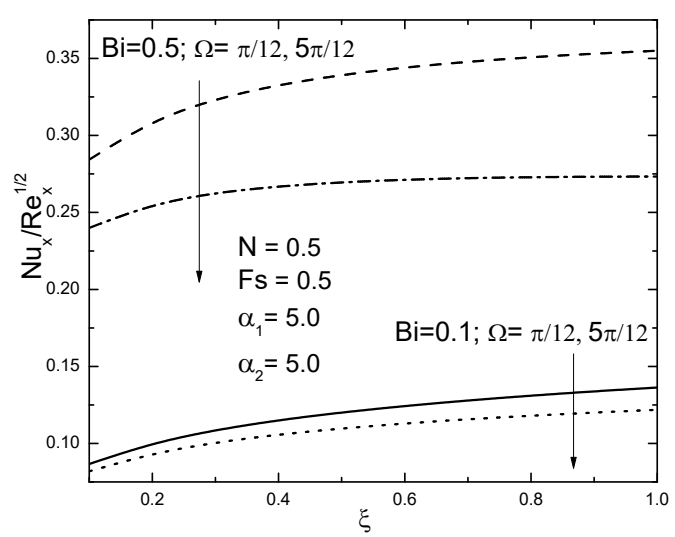

(a)

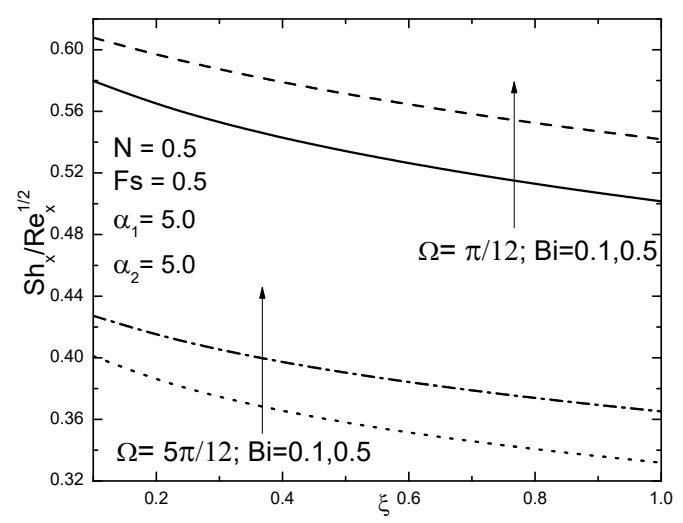

(b)

Fig. 8 Effect of Bi and $\Omega$ on the (a) Nusselt (b) Sherwood number's.

when compared with the NDC parameter effect. The insignificant influence of the wall couple stress and considerable increment in skin friction is experienced for high enough values of Biot number. Furthermore, the coefficient of drag and the wall couple stress reduce, as Forchheimer parameter increases.

\section{CONCLUSIONS}

A combination of the local non-similarity approach and successive linearization technique is used to study the free convection boundary-layer flow of a micropolar fluid over a convectively heated inclined plate in a saturated non-Darcy porous medium. The effects of various parameters on the velocity, microrotation, temperature, concentration, heat and mass transfer, skin friction and wall couple stress have been analyzed. The main findings are summarized as follows:

- The major conclusion is that the influence of nonlinear densityconcentration (NDC) parameter is additional outstanding on all the physical characteristics of the present model, compared therewith of nonlinear density-temperature (NDT) parameter.

- One of the implications of this study is that the mass and heat transfer rates of micropolar fluids are less than Newtonian fluid.

- It is perceived that the rise of the Biot number elevates the skin friction, mass, and heat transfer rates.

- Further, the velocity, microrotation, local mass and heat transfer rates reduce whereas, the boundary layer thicknesses of temperature and concentration enhance with the increase of inclination of angle. 
Table 3 Effect of skin friction and wall couple stress for various values of $N, F s, \alpha_{1}, \alpha_{2}, \Omega, B i$.

\begin{tabular}{|c|c|c|c|c|c|c|c|}
\hline$N$ & $F s$ & $\alpha_{1}$ & $\alpha_{2}$ & $\Omega$ & $B i$ & $f^{\prime \prime}(\xi, 0)$ & $-g^{\prime}(\xi, 0)$ \\
\hline 0.5 & 0.5 & 5.0 & 5.0 & 30 & 0.5 & 1.09565723 & 0.20210076 \\
0.0 & 0.5 & 5.0 & 5.0 & 30 & 0.5 & 1.70980700 & 0.00000000 \\
0.3 & 0.5 & 5.0 & 5.0 & 30 & 0.5 & 1.36342605 & 0.08611371 \\
0.8 & 0.5 & 5.0 & 5.0 & 30 & 0.5 & 0.58004739 & 0.52444524 \\
0.5 & 0.0 & 5.0 & 5.0 & 30 & 0.5 & 1.10628598 & 0.20474609 \\
0.5 & 0.8 & 5.0 & 5.0 & 30 & 0.5 & 1.08951988 & 0.20055820 \\
0.5 & 2.0 & 5.0 & 5.0 & 30 & 0.5 & 1.06664851 & 0.19471480 \\
0.5 & 0.5 & 0.0 & 5.0 & 30 & 0.5 & 0.99213184 & 0.19086334 \\
0.5 & 0.5 & 1.5 & 5.0 & 30 & 0.5 & 1.02420439 & 0.19438906 \\
0.5 & 0.5 & 5.5 & 5.0 & 30 & 0.5 & 1.10551683 & 0.20314992 \\
0.5 & 0.5 & 5.0 & 0.0 & 30 & 0.5 & 0.52178837 & 0.12870159 \\
0.5 & 0.5 & 5.0 & 3.0 & 30 & 0.5 & 0.87641989 & 0.17646774 \\
0.5 & 0.5 & 5.0 & 6.0 & 30 & 0.5 & 1.20123955 & 0.21367778 \\
0.5 & 0.5 & 5.0 & 5.0 & 15 & 0.5 & 1.18829140 & 0.21320555 \\
0.5 & 0.5 & 5.0 & 5.0 & 45 & 0.5 & 0.94213199 & 0.18277058 \\
0.5 & 0.5 & 5.0 & 5.0 & 75 & 0.5 & 0.44302379 & 0.10818973 \\
0.5 & 0.5 & 5.0 & 5.0 & 30 & 0.1 & 0.95972062 & 0.18510996 \\
0.5 & 0.5 & 5.0 & 5.0 & 30 & 5.0 & 1.46554157 & 0.24361328 \\
0.5 & 0.5 & 5.0 & 5.0 & 30 & 15 & 1.54705183 & 0.25227221 \\
\hline
\end{tabular}

\section{ACKNOWLEDGEMENT}

This work was supported by Council of Scientific and Industrial Research (CSIR), New Delhi, India (Project No 25 (0246)/15 /EMR-II).

\section{REFERENCES}

Awad, F., Sibanda, P., Motsa, S.S., and Makinde, O.D., 2011, "Convection from an inverted cone in a porous medium with cross-diffusion effects," Computers \& Mathematics with Applications, 61(5), 1431-1441. https://doi.org/10.1016/j.camwa.2011.01.015.

Aziz, A., 2009, "A similarity solution for laminar thermal boundary layer over a flat plate with a convective surface boundary condition," Cотmunications in Nonlinear Science and Numerical Simulation, 14(4), 10641068.

https://doi.org/10.1016/j.cnsns.2008.05.003.

Barrow, H., and Sitharamarao, T., 1971, "Effect of variation in volumetric expansion coefficient on free convection heat transfer," British Chemical Engineering, 16(8), 704-709.

Bég, O.A., Uddin, M., Rashidi, M., and Kavyani, N., 2014, "Doublediffusive radiative magnetic mixed convective slip flow with Biot and Richardson number effects," Journal of Engineering Thermophysics, 23(2), 79-97.

https://link.springer.com/article/10.1134/S1810232814020015.

Bég, O., Bhargava, R., Rawat, S., and Kahya, E., 2008, "Numerical study of micropolar convective heat and mass transfer in a non-Darcy porous regime with Soret and Dufour diffusion effects," Emirates J Eng Res, 13(2), 51-66.

Bejan, A., 2013, Convection heat transfer, John wiley \& sons.

Canuto, C., Hussaini, M.Y., Quarteroni, A., and Zang, T.A., 2006, Spectral Methods Fundamentals in Single Domains, Scientific Computation, Springer, Berlin, Germany.
Chamkha, A.J., Issa, C., and Khanafer, K., 2002, "Natural convection from an inclined plate embedded in a variable porosity porous medium due to solar radiation," International Journal of Thermal Sciences, 41(1), 73-81.

https://doi.org/10.1016/S1290-0729(01)01305-9.

Chen, C.H., 2004, "Heat and mass transfer in MHD flow by natural convection from a permeable, inclined surface with variable wall temperature and concentration," Acta Mechanica, 172(3), 219-235.

https://link.springer.com/article/10.1007/s00707-004-0155-5.

Cowin, S., 1968, "Polar Fluids," Physics of Fluids, 11, 1919-1927.

http://dx.doi.org/10.1063/1.1692219.

Eremeyev, V., Lebedev, L., and Altenbach, H., 2013, Foundations of Micropolar Mechanics, Springer, New York.

Eringen, A., 1966, "Theory of Micropolar Fluids," J Math and Mech, 16, $1-18$.

http://dx.doi.org/10.1512/iumj.1967.16.16001.

Kameswaran, P.K., Sibanda, P., Partha, M.K., and Murthy, P.V.S.N., 2014, "Thermophoretic and Nonlinear Convection in Non-Darcy Porous Medium," Journal of Heat Transfer, 136(4), Article ID 042601, 9 pages. http://dx.doi.org/10.1115/1.4025902.

Khidir, A.A., Narayana, M., Sibanda, P., and Murthy, P., 2015, "Natural convection from a vertical plate immersed in a power-law fluid saturated non-Darcy porous medium with viscous dissipation and Soret effects," Afrika Matematika, 26(7-8), 1495-1518.

Lukaszewicz, G., 1999, Micropolar fluids - Theory and Applications, Springer, Birkhaauser, Basel, Switzerland.

Makinde, O., 2010, "On MHD heat and mass transfer over a moving vertical plate with a convective surface boundary condition," The Canadian Journal of Chemical Engineering, 88(6), 983-990.

http://dx.doi.org/10.1002/cjce.20369 .

Makinde, O.D., and Aziz, A., 2011, "Boundary layer flow of a nanofluid past a stretching sheet with a convective boundary condition," International Journal of Thermal Sciences, 50(7), 1326-1332.

https://doi.org/10.1016/j.ijthermalsci.2011.02.019.

Makukula, Z.G., Sibanda, P., and Motsa, S.S., 2010, "A novel numerical technique for two-dimensional laminar flow between two moving porous walls," Mathematical Problems in Engineering, Article ID 528956, 15 pages.

http://dx.doi.org/10.1155/2010/528956.

Murthy, P., and Singh, P., 1999, "Heat and mass transfer by natural convection in a non-Darcy porous medium," Acta Mechanica, 138(3-4), 243254.

https://link.springer.com/article/10.1007/BF01291847.

Nield, D.A., and Bejan, A., 2013, Convection in Porous Media, Springer.

Partha, M.K., 2010, "Nonlinear Convection in a Non-Darcy Porous Medium," Applied Mathematics and Mechanics, 31, 565-574. http://dx.doi.org/10.1007/s10483-010-0504-6.

RamReddy, C., Pradeepa, T., and Srinivasacharya, D., 2015, "Similarity Solution for Free Convection Flow of a Micropolar Fluid under Convective Boundary Condition Via Lie Scaling Group Transformations," Advances in High Energy Physics, 2015, 1-16.

http://dx.doi.org//10.1155/2015/650813. 
Ramzan, M., Farooq, M., Hayat, T., and Chung, J.D., 2016, "Radiative and Joule heating effects in the MHD flow of a micropolar fluid with partial slip and convective boundary condition," Journal of Molecular Liquids, 221, 394-400.

https://doi.org/10.1016/j.molliq.2016.05.091.

Shaw, S., Kameswaran, P.K., and Sibanda, P., 2016, "Effects Of Slip on Nonlinear Convection in Nanofluid Flow on Stretching Surfaces," Boundary Value Problems, 2016(2).

http://dx.doi.org/10.1186/s13661-015-0506-2.

Sparrow, E., and Yu, H., 1971, "Local non-similarity thermal boundarylayer solutions," Journal of Heat Transfer, 93(4), 328-334.

Srinivasacharya, D., and RamReddy, C., 2013, "Free Convective Heat and Mass Transfer in a Doubly Stratified Non-Darcy Micropolar Fluid," Korean Journal of Chemical Engineering, 28(9), 1824-1832. http://dx.doi.org/10.1007/s11814-011-0069-6.

Vajravelu, K., and Sastri, K., 1977, "Fully developed laminar free convection flow between two parallel vertical walls-I," International Journal of Heat and Mass Transfer, 20(6), 655-660.

https://doi.org/10.1016/0017-9310(77)90052-7.

Yacob, N.A., and Ishak, A., 2012, "Stagnation Point Flow Towards Stretching/Shrinking Sheet in a Micropolar Fluid with a Convective Boundary Condition," Canadian Journal of Chemical Engineering, 90, 621-626.

http://dx.doi.org/10.1002/cjce.20517. 